\title{
Drug and alcohol misuse in first episode psychosis:An observational study
}

\author{
Kathleen Crebbin \\ Emma Mitford \\ Roger Paxton \\ Douglas Turkington \\ PACE Project, St George's Park, \\ Morpeth, Northumberland, UK
}

Correspondence: Emma Mitford PACE Project, St George's Park, Morpeth, Northumberland, UK NE6I 2NU

Tel +44 I670 50I 788

Fax +44 I670 50I 893

Email emma.mitford@ntw.nhs.uk
Background: There have been very few observational studies of drug and alcohol misuse in first-episode psychosis in the UK.

Method: Using an observational database of first episode psychosis in Northumberland, a county in Northern England, information on patients aged 16 to 36 years were collected at presentation and annual follow-up between October 1998 and October 2005. Patterns of drug and alcohol misuse were compared using hospitalization as an outcome measure, and violence rates were examined retrospectively.

Results: Drug misuse without alcohol misuse was associated with a highly significant increase in hospital days. An alcohol problem, either with or without coexisting drug misuse, was not predictive of increased hospital days. Drug and alcohol misuse together was associated with violence.

Conclusions: This paper lends some support to those Early Intervention in Psychosis (EIP) teams currently advising patients that drug misuse may have a greater impact than alcohol use on the outcome of first-episode psychosis.

Keywords: drug misuse, alcohol, early intervention, psychosis

\section{Introduction}

Comorbid substance misuse and psychosis have been shown to be associated with longer in-patient stays (Menezes et al 1996), increased symptom severity (Drake and Wallach 1989; Drake et al 1989), greater noncompliance with treatment (Bebbington 1995; Olfson et al 2000), higher rates of homelessness (Drake et al 1991) and increased aggression (Soyka 2000). A study by Barnett and colleagues (2007) found substance misuse in people with first episode psychosis to be twice that of the general population. Despite these associations, there is a paucity of evidence on the epidemiology of comorbid illegal drug misuse and alcohol misuse in first-episode psychosis.

Most research into the role of substance misuse in psychosis has focused on cannabis and amphetamines, and very little has been published in recent years on the role of alcohol misuse in psychosis. Although there seems little doubt that cannabis plays an important role in the development of schizophrenia, the relationship between alcohol and psychosis remains unclear. There is general agreement within the literature that aside from nicotine, alcohol and cannabis are the substances most commonly used by people with psychotic disorders (Margolese et al 2004). In a general adult population survey in Great Britain, alcohol dependence was predictive of psychotic experience (Johns et al 2004). The reported two-fold higher risk was independent of other risk factors for psychotic symptoms including drug dependence, suggesting that alcohol dependence per se doubles the risk of developing psychotic symptoms. There is also much evidence that substance misuse acts as a perpetuating factor for psychotic symptoms and, in fact, a reduction in substance misuse after diagnosis has been associated with a reduction in psychotic symptoms and hospital admissions (Lambert et al 2005). 
Most previous studies have used samples that included patients with varying durations of illness (Duke et al 1994; Menezes et al 1996; Graham et al 2001; McCreadie 2002; Weaver et al 2003; Wright et al 2000; Cantwell 2003). In fact, only 3 UK studies were found that focused on drug and alcohol misuse in first-episode psychosis (Cantwell et al 1999; Barnes et al 2006; Barnett et al 2007). In 1993, Cantwell pointed out that studies that focused on first-episode psychosis were almost exclusively North American, and this has not changed.

Most UK studies on first-episode psychosis published to date have concentrated on inner-city samples, particularly London (Duke et al 1994; Menezes et al 1996; Barnes et al 2006). Only two UK studies (McCreadie 2002; Cantwell 2003) and one US study included patients from urban and rural settings.

The UK government has invested heavily over the past decade in developing Early Intervention in Psychosis (EIP) teams as an integral part of standard mental health services across the country (Pelosi and Birchwood 2003). The aim of EIP teams is, in line with British Health Policy, to provide quick and effective treatment to patients aged under 36 years with the rationale that early treatment of psychotic symptoms leads to a higher success rate. The objectives of the study were to examine the effects of drug and alcohol misuse in a sample of 251 people aged under 36 years who presented with a first episode psychosis between October 1998 and October 2005 drawn from rural and urban settings in a geographically defined region in the north of England. Prospective data on hospital admissions and retrospective data on rates of violence in the substance misuse groups were analyzed.

\section{Method}

\section{Background}

The PACE (population-adjusted clinical epidemiology) process was developed and refined over a 15 year period in hematological malignancy (Charlton et al 1997; Proctor and Taylor 2000; Proctor 2002). In 1998 the methodology was adapted for use in mental health. PACE is an observational database used to record prospective data on the presentation, management, and outcomes of all patients aged 16 years and over with a first-episode psychosis referred to secondary care mental health services in a Northumberland, a county in northern England. It was developed for the purposes of service evaluation and audit, with the aim of improving services (Proctor et al 2004). For example, a recent PACE study (Crebbin et al 2007) found a surprisingly high incidence of psychotic depression that will lead the Trust to review its policies and procedures for this patient group.

\section{Participants}

Data on patients aged between 16 and 36 years (at the time of first presentation) seen by adult consultant psychiatrists in Northumberland between 1st October 1998 and 30th September 2005 with a first episode psychotic illness, according to ICD-10 criteria, were included. The ICD-10 diagnosis definitions used in this study are shown in Table 1. The only exclusion criteria were: presentation outside of the Trust area with a first-episode psychosis and a past history of treated

Table I ICDIO diagnoses and definitions used for PACE

\begin{tabular}{|c|c|c|}
\hline ICDIO code & Diagnosis & $\begin{array}{l}\text { No. of patients } \\
\text { under } 36 \text { years } \\
(n=25 I)\end{array}$ \\
\hline F6.2 & Organic delusional (schizophrenia-like) disorder & 4 \\
\hline FIO-19.5 & $\begin{array}{l}\text { Mental and behavioral disorders due to use of psychoactive } \\
\text { substance use with psychotic symptoms }\end{array}$ & $35(2$ with FI0.5) \\
\hline $\mathrm{F} 20$ & Schizophrenia & 42 \\
\hline F2I & Schizotypal disorder & 0 \\
\hline $\mathrm{F} 22$ & Persistent delusional disorders & 5 \\
\hline F23 & Acute and transient psychotic disorders & 62 \\
\hline $\mathrm{F} 25$ & Schizoaffective disorders & 7 \\
\hline F28 & Other nonorganic psychotic disorders & 45 \\
\hline F29 & Unspecified nonorganic psychosis & 2 \\
\hline $\mathrm{F} 30.2$ & Manic episode with psychotic symptoms & 5 \\
\hline $\mathrm{F} 31.2$ & Bipolar affective disorder with psychotic symptoms & 13 \\
\hline F32.3 & Severe depressive episode with psychotic symptoms & 31 \\
\hline F20-29 & Schizophrenia, schizotypal, and delusional disorders & 163 \\
\hline F30-33 & Mood (affective) disorders & 49 \\
\hline
\end{tabular}


psychosis. Information on patients' drug and alcohol misuse patterns were collected from secondary care medical notes at the time of presentation to secondary care services. This data relied on clinician judgment and patients' self-reports. Patients were subsequently divided into four groups:

1. Illegal drug misuse. This included patients who were currently using any illegal drugs regardless of amount or frequency.

2. Alcohol misuse. This included patients reported in case notes to have an alcohol problem at the time of presentation, or who consumed above the Government's recommended weekly limits.

3. Both drug and alcohol misuse. This included patients who were reported as using illegal drugs and having an alcohol problem.

4. Neither drug or alcohol misuse. These patients were not reported as using illegal drugs or misusing alcohol.

\section{Procedure}

Patient records for all patients with a first episode psychosis presenting to the Trust between 1st October 1998 and 30th September 2005 were examined by PACE staff and relevant data were extracted. Information regarding demographics, drug misuse, alcohol misuse, hospital admissions, and risk of violence were collected from inpatient and outpatient secondary care medical notes and the data were entered onto an Access database. Patients' secondary care medical notes were searched annually on the anniversary of their presentation. Monthly reminders were sent to all consultant psychiatrists, asking for names of patients who had recently presented with a first-episode psychosis, both inpatients and outpatients. PACE staff also screened the computerized patient information system for potential patients who had been discharged from hospital.

\section{Analysis}

On the basis of outcome data participants were categorized into one of the four substance misuse groups and the data were analyzed using SPSS 14.0 for Windows. Continuous data were analysed using either an analysis of variance test or independent samples t-test, and the ordinal data using the chi-square test. Differences significant at least at the 5\% level were reported.

\section{Confidentiality}

Confidentiality was strictly maintained. After data collection, patient identifiable data were stored on a secure database, and in a locked cabinet, which only PACE staff were able to access. For the purposes of analysis, patients were given unique PACE identification numbers and data were always presented in the form of group outcomes. PACE staff had no contact with patients, and were not involved in their care or management. The database was set up with the aim of service evaluation as defined by the National Research Ethics Service (see www.nres.npsa.nhs.uk), therefore, did not require ethical approval.

\section{Results \\ Characteristics of the sample}

251 patients aged under 36 years presented between October 1998 and October 2005 with a first episode psychosis, diagnosed according to ICD-10 criteria. There were 176 males and 75 females with a mean age of 25.1 years [standard deviation (SD) 5.6 years]. The ages ranged from 16 to 35 years.

At presentation, 140 (55.8\%) currently used illicit drugs (with or without alcohol) and 88 (35.1\%) currently misused alcohol (with or without drugs). For comparison, this sample was divided into 4 groups:

1. Illicit drug misuse $(\mathrm{n}=77$; mean age 23.3 years; SD 5.4 years). This group included 60 males and 17 females $[$ Risk ratio $(\mathrm{RR})=0.567$; Confidence interval $(\mathrm{CI})=0.304,1.058]$.

2. Alcohol misuse $(n=25$; mean age 25.6 years; SD 5.9 years). This group included 16 males and 9 females $(\mathrm{RR}=1.364 ; \mathrm{CI}=0.574,3.24)$.

3. Both illicit drug misuse and alcohol misuse $(n=63$; mean age 24.4 years; SD 5.4 years). This group included 55 males and 8 females $(\mathrm{RR}=0.263 ; \mathrm{CI}=0.118,0.584)$.

4. No illicit drug misuse or alcohol misuse $(n=86$; mean age 27.0 years; SD 5.4 years). This group included 45 males and 41 females $(\mathrm{RR}=3.510 ; \mathrm{CI}=1.991,6.188)$.

\section{Hospital admissions}

All diagnoses $(n=251)$

A one-way between groups analysis of variance (ANOVA) was conducted to explore differences in hospital days between the groups. Table 2 shows hospital admission data for the groups. There was a statistically significant difference at the $\mathrm{p}<0.01$ level between the four groups for hospital days $[F(3,247)=4.8, \mathrm{p}=0.003]$. Post-hoc comparisons using the Tukey HSD test indicated that patients who misused drugs had significantly more hospital days than those who: misused alcohol [mean of 210 days (SD 393) compared with 36 days ( $\mathrm{SD} 87) ; \mathrm{p}=0.02]$, misused both drugs and alcohol [mean of 76 days (SD 171); $\mathrm{p}=0.02$ ], and did not misuse drugs or alcohol [mean of 95 days (SD 193); $\mathrm{p}<0.03$ ]. No 
Table 2 Risk assessment and hospital admissions

\begin{tabular}{llllll}
\hline All diagnoses & Total & Drugs & Alcohol & $\begin{array}{l}\text { Both drugs } \\
\text { and alcohol }\end{array}$ & $\begin{array}{l}\text { No sub } \\
\text { misuse }\end{array}$ \\
\hline $\begin{array}{l}\text { Total number of patients } \\
\text { Violence towards others and property }\end{array}$ & 251 & 77 & 25 & 63 & 86 \\
$\begin{array}{l}\text { Violence to others } \\
\text { Violence to property }\end{array}$ & $63(25 \%)$ & $22(29 \%)$ & $5(20 \%)$ & $29(46 \%)$ & $7(8 \%)$ \\
$\begin{array}{l}\text { Hospital admissions } \\
\text { Number admitted }\end{array}$ & $47(19 \%)$ & $19(25 \%)$ & $2(8 \%)$ & $22(35 \%)$ & $4(5 \%)$ \\
Number of days & 155 & 51 & 9 & 45 & 50 \\
Mean days (SD) & 30043 & 16171 & 896 & 4786 & 8190 \\
\hline
\end{tabular}

significant differences were found between patients who misused alcohol and those who did not misuse any substances $(\mathrm{p}=0.97)$.

The covariate effect of the substance misuse groups on the total number of hospital days was significant at $F(1,248)=7.48, \mathrm{p}=0.007$.

\section{Violence towards others and property} All diagnoses $(n=25 \mathrm{I})$

A chi-square test was conducted to explore differences between the groups regarding physical violence towards others and violence towards property measured at the time of first presentation to services. Table 2 shows rates of violence for the groups. Patients who misused both drugs and alcohol were more likely $(p<0.001)$ to have been physically violent towards others prior to presentation $(n=29 ; 46 \%)$ than those who: misused drugs $(\mathrm{n}=22 ; 29 \%)$, misused alcohol $(\mathrm{n}=5$; $20 \%$ ), and did not misuse substances ( $\mathrm{n}=7 ; 8 \%)$.

Patients who misused both drugs and alcohol were also more likely $(\mathrm{p}<0.001)$ to have harmed property $(\mathrm{n}=22$; $35 \%)$ than those who misused drugs $(\mathrm{n}=19 ; 25 \%)$, misused alcohol $(\mathrm{n}=2 ; 8 \%)$, and did not misuse substances $(\mathrm{n}=4 ; 5 \%)$.

Of the 251 patients, only 159 (63\%) were still in contact with secondary care mental health services after 1 year. Of those, 46 (29\%) still had a documented alcohol problem, 44 (28\%) were still misusing drugs and 19 (12\%) had a drug and alcohol problem. Over the previous year, 19 (12\%) had physically harmed others. We will be investigating this further. There is no information available on what has happened to those who are no longer in contact with psychiatric services.

\section{Discussion}

\section{Prevalence and characteristics of the sample}

In line with previous findings, drug and alcohol misuse in people with first episode psychosis aged under 36 years was high (Hambrecht and Hafner 1996; Barnes et al 2006; Barnett et al 2007). Over half of our sample were misusing drugs at presentation, and over a third had an alcohol problem. Although it is difficult to compare our rates with those of previous studies, due to differences in samples and methodologies, it seems the rates for drug and alcohol misuse in our study are a little higher than those reported in previous UK studies (Duke et al 1994; Menezes et al 1996; Cantwell et al 1999; McCreadie et al 2002). It has been argued that the prevalence of drug misuse is higher in inner-cities, particularly inner-city London, than in suburban or rural areas (Weaver et al 2003; Barnes et al 2006). The current study was carried out in Northumberland, a county consisting of urban and rural areas. It is the least densely populated county in England, but over half of the population live in less than $5 \%$ of the total land area (see www.northumberland.gov.uk). It could be that patterns of drug and alcohol misuse differ between urban and rural environments, but not necessarily that there is less substance misuse in rural parts of the UK. In the US, Mueser and colleagues (2001) compared substance misuse in schizophrenia in a rural and an urban area and found the rural area had higher rates of alcohol and cannabis misuse, and lower rates of cocaine misuse. Weaver and colleagues (2003) reported substance misuse in patients with psychotic illnesses in a suburban area to be about as high as that for similar patients in inner-city London.

The present study supports previous research that has found a higher proportion of young male patients among substance misusers (Cantwell et al 1999; Barnes et al 2006). Age differences between the groups were not significant, although this will have been influenced by the fact that only patients under 36 years were included in this study.

\section{Hospital admission days}

A number of studies have found an association between increased hospital days and drug and alcohol misuse in 
patients with psychosis (Drake et al 1989; Menezes et al 1996; Swofford 1996), and others have found no association (Duke et al 2001; Cantwell 2003). We found that patients who misused drugs spent more days in hospital than those who misused both drugs and alcohol, and those who misused alcohol. Drug misuse was associated with more hospital days but alcohol misuse was not. No significant difference was found between patients who misused alcohol and those who did not misuse any substances. To our knowledge, no other studies have examined drug and alcohol misuse separately, and most have combined drug and alcohol misuse and studied substance misuse (Cantwell et al 2003).

Modestin and colleagues (2001) who studied dual diagnosis in schizophrenic patients concluded that patients who misused illegal drugs had poorer outcomes than those who misused alcohol and those who did not misuse substances. Duke and colleagues (1994) found that patients who misused alcohol had a shorter length of illness than those who did not. They suggested the possibility that alcohol may protect patients from presenting in the early stages possibly by alleviating the distress associated with acute illness. They also suggested that alcohol may have masked the early stages of a psychotic illness so that later age at onset and shorter length of illness were recorded in their study. Duke and colleagues (1994) and Modestin and colleagues (2001) found the course of psychotic illness in patients who misused alcohol to be similar to that in those who did not misuse any substances. Our results support this finding and no difference was found with regard to overall hospital days between patients who misused alcohol and those who did not misuse any substances.

\section{Violence towards people and property}

Although patients who misused drugs spent more days in hospital than all other groups, those who misused both drugs and alcohol were significantly more likely to have been violent towards other people and property. This supports previous evidence that patients with schizophrenia who misused alcohol were more likely to commit violent offences than those who did not misuse alcohol (Swanson et al 1990; Rasanen et al 1998). A consistent relationship has been demonstrated between alcohol misuse and violent behavior in the general population. Swanson (1993) argued that although not all alcoholics are violent, they are more likely than nonalcoholics to have a history of violent behavior. A number of reasons have been suggested to explain the relationship between alcohol and violence, including biochemical, psychological, cultural, and situational factors. Higher rates of violence and aggression among people with mental health disorders have repeatedly been shown, particularly when they misused drugs (Soyka 2000). A literature search did not reveal any studies that compared the effects of drugs and alcohol separately on violence in people with psychosis. Further research with larger samples is needed to understand the complex relationship between psychosis, drugs, alcohol, and violence.

\section{Strengths and weaknesses}

A unique strength of this study is that it used 8 years of prospective follow-up data, and data were gathered from a defined catchment area with a relatively stable population. Another strength is that very few cases of first-episode psychosis were missed. Data were collected on patients under 36 who were seen by the Northumberland EIP team or were referred to the PACE team by Consultant Psychiatrists. Data on all people who had a hospital admission were collected and this was checked through the Trust's computerized patient information system.

The fact that all first-episode psychosis diagnoses were included meant that the present study was more representative of all cases of first-episode psychosis in people aged under 36 years who would be referred to an EIP team, as diagnoses of substance-induced psychoses and affective psychoses were included. Most UK studies have focused on schizophrenia and schizophrenia-spectrum disorders and have excluded other psychotic diagnoses (Duke et al 2001; McCreadie 2002; Cantwell 2003; Barnes et al 2006; ). Furthermore, the present study included inpatients and outpatients who had accessed secondary mental health services for treatment for a first-episode of psychosis. Therefore, data were also collected for patients who had not been admitted to hospital. Many previous studies of substance misuse in first-episode psychosis have been defined by first admission (Hambrect and Hafner 1996; Barnes et al 2006). It is arguable that these fail to reflect accurately the rates of comorbidity by excluding those who have not been admitted to hospital. In patients with psychosis, those receiving in-patient care have been shown to have higher rates of comorbidity of drug misuse (Mueser et al 1990). The observational design used in the present study is more likely to reflect the true prevalence and course of substance misuse among people with first-episode psychosis.

The PACE methodology does have limitations. There was no detailed information about the frequency and amounts of drugs taken, nor the degree of dependence. However, even relatively low levels of comorbid substance misuse appear to be associated with poor outcomes in schizophrenia, including 
a greater risk of relapse and poorer response to conventional antipsychotics (Kavanagh et al 2004; Barnes et al 2006). Inclusion in the alcohol misuse category was more stringent than for drug misuse as patients were only included if clinicians had reported that they had an alcohol problem, whereas any use of illicit drugs led to inclusion in the drug misuse category. A common problem, which we share with other studies, is the possible unreliability of self-reports of drug and alcohol misuse. However, a number of studies have recently found patients' self-reported drug misuse to be accurate and to correlate highly with urine drug-screening (Hides et al 2006).

Some confounding variables that we did not measure or control for may have influenced the results of this study, such as socio-economic or employment factors. Variables such as male gender, young age, substance misuse, and violence which have been closely linked in previous studies (Soyka 2000) may have confounded some findings.

A further limitation was the small sample sizes, particularly for the alcohol misuse group $(n=25)$. This study needs to be replicated with a larger sample before conclusions about the effects of alcohol, either on its own or in combination with drugs, can be made.

\section{Implications for the future}

In first episode psychosis, a co-existing alcohol problem does not appear to lead to poorer outcomes, as measured by hospital days. Many EIP teams are currently advising patients that using "skunk" cannabis, amphetamine, and cocaine may be more harmful to them than alcohol. Although this strategy is controversial, this report provides some support for it. Further research and replication of these findings are needed. A future study should include a larger sample size and the collection of potential confounding variables, such as socio-economic and employment factors, which would allow an analysis of covariates.

Our findings support Barnett and colleagues (2007) who recommended that close cooperation is needed between EIP teams and substance misuse services. These findings have implications for staff training. EIP teams should be aware of the higher risk of violence towards others associated with a combination of alcohol and drugs, and drug and alcohol intake reduction strategies should be a priority.

\section{Conclusions}

High rates of drug and alcohol misuse in young people with first-episode psychosis highlight the need for the development of effective therapeutic interventions which address drug and alcohol misuse and violent behavior in this high-risk group. Drug and alcohol histories should be properly detailed by clinicians to help further case-note analyses, and to enable further research.

\section{References}

Barnes TRE, Mutsatsa SH, Hutton SB, et al. 2006. Comorbid substance use and age of onset of schizophrenia. Br J Psychiatry, 188:237-42.

Barnett JH, Werners U, Secher SM, et al. 2007. Substance use in a population-based clinic sample of people with first-episode psychosis. $\mathrm{Br} J$ Psychiatry, 190:515-20.

Bebbington PE. 1995. The content and context of compliance. Int Clin Psychopharmacol, 9:41-50.

Cantwell R, Brewin J, Glazebrook C, et al. 1999. Prevalence of substance misuse in first-episode psychosis. Br J Psychiatry, 174:150-3.

Cantwell R. 2003. Substance use and schizophrenia: effects on symptoms, social functioning and service use. Br J Psychiatry, 182:324-9.

Charlton B, Taylor PRA, Proctor SJ. 1997. The PACE strategy: a new approach to multicentre clinical research. Quart J Med, 90:147-51.

Crebbin K, Mitford E, Paxton R, et al. 2007. First-episode psychosis: An epidemiological survey comparing psychotic depression with schizophrenia. $J$ Affect Disord, Jun 13 [In press].

Drake RE, Wallach MA. 1989. Substance abuse among the chronic mentally ill. Hosp Comm Psychiatry, 40:1041-6.

Drake RE, Osher FC, Wallach MA. 1989. Alcohol use and abuse in schizophrenia. J Nerv Ment Dis, 17:408-14.

Drake RE, Osher FC, Wallach MA. 1991. Homelessness and dual diagnosis. Am Psychol, 46:1149-58.

Duke PJ, Pantelis C, Barnes TRE. 1994. South Westminster schizophrenia survey. Alcohol use and its relationship to symptoms, tardive dyskinesia and illness onset. Br J Psychiatry, 164:630-6.

Duke PJ, Pantelis C, McPhillips MA, et al. 2001. Comorbid non-alcohol substance misuse among people with schizophrenia. Epidemiological study in central London. Br J Psychiatry, 179:509-13.

Graham HL, Maslin J, Copello A, et al. 2001. Drug and alcohol problems among individuals with severe mental health problems in an inner city area of the UK. Soc Psychiatry Psychiatr Epidemiol, 36:448-55.

Hambrecht M, Hafner H. 1996. Substance abuse and the onset of schizophrenia. Biol Psychiatry, 40:1155-63.

Hides L, Dawe S, Kavanagh DJ, et al. 2006. Psychotic symptom and cannabis relapse in recent-onset psychosis. Br J Psychiatry, 189:137-43.

Johns LC, Cannon M, Singleton N, et al. 2004. Prevalence and correlates of self-reported psychotic symptoms in the British population. $\mathrm{Br} J$ Psychiatry, 185:298-305.

Kavanagh DJ, Waghorn G, Jenner L, et al. 2004. Demographic and clinical correlates of comorbid substance use disorders in psychosis: multivariate analyses from an epidemiological sample. Schizophr Res, 66:115-24.

Margolese HC, Malchy L, Negrete JC, et al. 2004. Drug and alcohol use among patients with schizophrenia and related psychoses: levels and consequences. Schizophr Res, 67:157-66.

Modestin J, Studer Gladen CJ, Christen S. 2001. A comparative study on schizophrenic patients with dual diagnosis. J Addict Dis, 20:41-51.

McCreadie RG. 2002. Use of drugs, alcohol and tobacco by people with schizophrenia: case-control study. Br J Psychiatry, 181:321-5.

Menezes PR, Johnson S, Thornicroft G, et al. 1996. Drug and alcohol problems among individuals with severe mental illness in south London. $\mathrm{Br}$ J Psychiatry, 168:612-19.

Mueser KT, Essock SM, Drake RE, et al. 2001. Rural and urban differences in patients with a dual diagnosis. Schizophr Res, 48:93-107.

Olfson M, Mechanic D, Hansell S, et al. 2000. Predicting mdication nncompliance after hospital discharge among patients with schizophrenia. Psychiatr Serv, 51:216-22.

Pelosi AJ, Birchwood M. 2003. Is early intervention for psychosis a waste of valuable resources. Br J Psychiatry, 182:196-8. 
Proctor SJ. 2002. The use of population-based research approaches in haematological malignancy: a general medical research tool. In: Amiel S (ed). Horizons in Medicine: Number 13, 1st ed. London: Royal College of Physicians, pp. 219-26.

Proctor SE, Mitford E, Paxton R. 2004. First episode psychosis: a novel methodology reveals higher than expected incidence; a realitybased population profile in Northumberland, UK. J Eval Clin Pract, 10:539-47.

Rasanen P, Tiihonen J, Isohanni M, et al. 1998. Schizophrenia, alcohol abuse, and violent behavior: a 26-year follow-up study of an unselected birth cohort. Schizophr Bull, 24:347-441.

Soyka M. 2000. Substance misuse, psychiatric disorder and violent and disturbed behavior. Br J Psychiatry, 176:345-50.
Swanson JW. 1993. Alcohol abuse, mental disorder, and violent behavior: An epidemiological inquiry. Alcohol Health Res World, 17:123-32.

Swofford C. 1996. Substance use: a powerful predictor of relapse in schizophrenia. Schizophr Res, 20:145-51.

Weaver T, Madden P, Stimson VCG, et al. 2003. Comorbidity of substance misuse and mental illness in community mental health and substance misuse. Br J Psychiatry, 183:304-13.

Wright S, Gournay K, Glorney E, et al. 2000. Dual diagnosis in the suburbs: prevalence, need, and in-patient service use. Soc Psychiatry Psychiatr Epidemiol, 35:297-304. 
